\title{
Development of a Chiral Capillary Electrophoresis Method for the Enantioseparation of Verapamil Using Cyclodextrins as Chiral Selectors and Experimental Design Optimization
}

\author{
Melania Cârcu-Dobrin ${ }^{1, *+\dagger}$, Gabriel Hancu ${ }^{1,+}{ }^{\mathbb{D}}$, Lajos Attila Papp ${ }^{1} \mathbb{C}$, Ibolya Fülöp ${ }^{2}$ and Hajnal Kelemen ${ }^{1}$ \\ 1 Department of Pharmaceutical and Therapeutic Chemistry, Faculty of Pharmacy, University of Medicine, \\ Pharmacy, Science and Technology “George Emil Palade" of Târgu Mureș, 540142 Târgu Mureș, Romania; \\ gabriel.hancu@umfst.ro (G.H.); lajos.papp@umfst.ro (L.A.P.); hajnal.kelemen@umfst.ro (H.K.) \\ 2 Department of Toxicology and Biopharmacy, Faculty of Pharmacy, University of Medicine, Pharmacy, Science \\ and Technology "George Emil Palade" of Târgu Mureș, 540142 Târgu Mureș, Romania; ibolya.fulop@umfst.ro \\ * Correspondence: melania.carcu@umfst.ro \\ + These authors share the first authorship.
}

Citation: Cârcu-Dobrin, M.; Hancu, G.; Papp, L.A.; Fülöp, I.; Kelemen, H. Development of a Chiral Capillary Electrophoresis Method for the Enantioseparation of Verapamil Using Cyclodextrins as Chiral Selectors and Experimental Design Optimization. Symmetry 2021, 13, 2186. https://doi.org/10.3390/ sym 13112186

Academic Editor: P. Ulrich Biedermann

Received: 27 September 2021 Accepted: 12 November 2021 Published: 16 November 2021

Publisher's Note: MDPI stays neutral with regard to jurisdictional claims in published maps and institutional affiliations.

Copyright: (c) 2021 by the authors. Licensee MDPI, Basel, Switzerland. This article is an open access article distributed under the terms and conditions of the Creative Commons Attribution (CC BY) license (https:/ / creativecommons.org/licenses/by/ $4.0 /)$.
Abstract: Chirality is a property of asymmetry which determines the pharmacokinetic and pharmacological profiles of optically active pharmaceuticals. Verapamil (VER), a calcium channel blocker phenylalkylamine derivative used in the treatment of cardio-vascular diseases, is a chiral compound, marketed as a racemate, although differences between the pharmacokinetic and pharmacological attributes of the enantiomers have been reported. The aim of our study was to develop a new chiral separation method for VER enantiomers by capillary electrophoresis (CE) using cyclodextrins (CDs) as chiral selectors (CSs). After an initial screening, using different native and derivatized CDs, at four $\mathrm{pH}$ levels, heptakis 2,3,6-tri-O-methyl- $\beta$-CD (TM- $\beta-C D)$, a neutral derivatized CD, was identified as the optimum CS. For method optimization, a preliminary univariate approach was applied to characterize the influence of analytical parameters on the separation followed by a Box-Behnken experimental design to establish the optimal separation conditions. Chiral separation of enantiomers was achieved with a resolution of 1.58 in approximately $4 \mathrm{~min}$; the migration order was $R$-VER followed by $S$-VER. The method analytical performance was evaluated in terms of precision, linearity, accuracy, and robustness (applying a Plackett-Burnam experimental design). The developed method was applied for the determination of VER enantiomers in pharmaceuticals. Finally, a computer modelling of VER-CD complexes was used to describe host-guest chiral recognition.

Keywords: verapamil; chiral separation; capillary electrophoresis; cyclodextrin; experimental design; molecular modelling

\section{Introduction}

It is known that symmetry and chirality have particular meanings in chemistry, especially when characterizing optically active pharmaceutical substances. Approximately 50\% of the substances currently used in therapy are chiral, having at least a center of asymmetry in their structure; leading to the existence of two or more enantiomers. The differences between the pharmacokinetic and pharmacological profiles of the enantiomers in the case of many chiral substances are already established, as some are used in therapy as pure enantiomers and others as racemic mixtures [1].

Verapamil (VER) [R,S-2-(3,4-dimethoxyphenyl)-5-[2-(3,4-dimethoxyphenyl)ethyl-methylamino]-2-propan-2-ylpentanenitrile] is a phenylalkylamine derivative commonly used in the treatment of supraventricular and ventricular arrhythmias, hypertension, and angina pectoris. It acts as a calcium channel blocking agent, being characterized by high selectivity for myocardial calcium channels and lower for the ones located in the vessels [2].

VER is administered in therapy as a racemate containing equivalent amounts of the $S$ and $R$-VER. $S$-VER is more potent as an antiarrhythmic than $R$-VER, but it is metabolized 
at a faster rate. Determinations of the pharmacokinetic profile of VER in human plasma showed that $R$-VER has a more than double the bioavailability than $S$-VER. Furthermore, $S$-VER has negative chronotropic and inotropic effects, producing systemic and coronary artery vasodilation, and slows atrioventricular node conduction, while $R$-VER, causes vasodilation of the arterial and coronary arteries but has few direct cardiac effects. VER undergoes extensive first-pass metabolism, mainly through demethylation, which is stereoselective, forming its main metabolite, norverapamil (NVER), as well as other several minor metabolites [3,4].

The chemical structure of VER enantiomers is presented in Figure 1.<smiles>[R1][R11]([R])=O</smiles>

Figure 1. Chemical structure of VER enantiomers.

Capillary electrophoresis (CE) is a modern, effective technique for the enantioseparation of pharmaceutical substances, the separation being accomplished usually by a direct approach by simply adding the chiral selector (CS) directly into the background electrolyte (BGE). Currently, CE is considered as an alternative to the most commonly used chromatographic methods for the chiral separation of pharmaceuticals. The main advantages of chiral CE are related to the high separation efficiency, relatively short analysis time and rapid method development. Moreover, this technique has significantly lower operational costs when compared to high performance liquid chromatography (HPLC), due to the low consumption of solvents, samples, and CSs; and it is also considered a more ecologically friendly analytical alternative $[5,6]$.

The most frequently used CSs, in CE, are the cyclodextrin (CD) derivatives (natural and derivatized, neutral, and ionized) due to their high complexing and interaction capacity with a large number of pharmaceutical substances, low UV absorption, and their wide commercial availability [7].

Traditionally, for the development and optimization of analytical methods, the univariate "one factor at a time" (OFAT) strategy is used, in which one analytical factor is varied while the others remain at a constant value [8]. In this study, we chose to apply an OFAT approach in the preliminary development of the method as an intermediate stage in the subsequent implementation of a more complex experimental design strategy for method optimization.

Modern strategies using design of experiments (DoE) involve establishing not only the influence of certain experimental factors on the studied analytical responses, but also the identification of complex relationships between experimental factors [8]. 
Several articles regarding CE chiral separation of VER have been published in the literature. Dethy et al. developed a CE chiral separation for the simultaneous determination of VER and NVER in human plasma, using trimethyl- $\beta-C D$ as CS [9]. Micellar electrokinetic capillary chromatography (MEKC), using mixed bile salts (sodium deoxycholate) and polyoxyethylene ethers, was used by Clothier \& Tomellini for the simultaneous base-line separation of verapamil, NVR, and gallopamil (a structural analogue) enantiomers [10]. Chankvetadze et al. studied the chiral recognition of VER enantiomers by CDs ( $\beta-C D$, heptakis [2,3,6-tri-O- methyl]- $\beta$-CD [TM- $\beta$-CD]) with CE, NMR spectroscopy, and electrospray ionization-mass spectrometry (ESI-MS) [11]. The enantioseparation of VER and NVER in human plasma was also obtained through CE by Resztak \& Glowka; TM- $\beta-\mathrm{CD}$ at $\mathrm{pH} 2.5$ was used as CS [12].

Another interesting study by Hamidi \& Jouyban applies a dispersive liquid-liquid microextraction (DLLME) method coupled with field-amplified sample injection (FASI) in $C E$, with UV detection for the quantification of VER enantiomers in human plasma. Separation was performed using carboxymethyl- $\beta-C D(C M-\beta-C D)$ as CS [13].

The enantioselective binding between VER and plasma lipoproteins was studied by Mohammed et al. using a CE method coupled with frontal analysis. It was found that VER binding to high-density lipoprotein (HDL), low-density lipoprotein (LDL), and oxidized LDL is non-enantioselective, with partition-like binding to the lipid part being dominant [14].

Ha et al., developed an in vitro CE method for the simultaneous separation of $R$ - and $S$-VER and their major metabolites, $R$ - and $S$-NVER, using sulfated $\beta$-CD (S- $\beta$-CD) as CS and studied an in-capillary enzymatic reaction between the substrate (VER) in a reaction mixture with CYP3A4, in the presence of NADP as a co-enzymatic factor. Based on the results, the conclusion was that no enantioconversion occurred and $R$-VER has a 1.5 higher metabolic rate than S-VER [15]. In another study, Asensi-Bernardi et al. performed an inline evaluation of the chiral particularities of VER metabolism by CE. The process included an electrophoretically induced in-capillary reaction step and a separation stage in which highly sulfated- $\beta$-cyclodextrin (HS- $\beta$-CD) with partial filling technique was used as a CS for the chiral resolution of VER and NVER enantiomers. Differences between CYP3A4 metabolisms of $R$ - and $S$-VER were identified [16].

The stereoselective biodegradation of VER and citalopram (a chiral selective serotonin reuptake inhibitor) was studied by Martin-Biosca et al. using CE and highly sulphated$\gamma$-cyclodextrin (HS- $\gamma$-CD) as CS. The biodegradability results showed that VER is not considered to be a persistent compound in the environment; in the biodegradation process, there was no evidence of enantioselectivity [17]. VER was used as a model chiral substance in testing amino acid chiral ionic liquids as dual CS systems with hydroxypropyl- $\beta$-CD (HP$\beta-C D)$ [18], stereoselectivity interactions between human serum albumin (HAS) [19], or a new on-capillary sample enrichment technique which leads to a sensitivity improvement in CE [20].

The aim of the current article is to develop a new, fast, and efficient CE method for the enantioresolution of VER enantiomers using CD as CS and applying a DoE approach in method optimization.

\section{Materials and Methods}

\subsection{Chemicals and Reagents}

A pharmaceutical-grade racemic mixture of VER hydrochloride (Piramal Enterprises Ltd., Hyderabad, India) and standard S-VER hydrochloride (Toronto Research Chemicals, Toronto, ON, Canada) was used as an analyte. The following reagents were used: phosphoric acid $85 \%$, disodium hydrogenophosphate (Merck, Darmstadt, Germany), monosodium hydrogen phosphate (Alfa Aesar, Karlsruher, Germany), sodium hydroxide (Lach-Ner, Neratovice, Czech Republic), and methanol (Merck, Darmstadt, Germany). Deionized water was prepared using a Milli-Q apparatus (Millipore, NH, USA). All reagents were of analytical grade. 
The following CDs were used as CS in the screening process: neutral natural CDs $\alpha-C D, \beta-C D$, and $\gamma-C D$ (Cyclolab, Budapest, Hungary); neutral derivatized CDs hydroxypropyl- $\beta-C D(H P-\beta-C D)$ (Sigma-Aldrich, Taufkirchen, Germany), methylated $\beta-C D(M-\beta-$ CD) (Cyclolab, Budapest, Hungary), heptakis(2,6-di-O-methyl)- $\beta-C D$ (DM- $\beta-C D)$, and heptakis(2,3,6-tri-O-methyl)- $\beta$-CD (TM- $\beta$-CD) (Sigma-Aldrich, Taufkirchen, Germany); and ionizable derivatized anionic CDs carboxymethyl- $\beta-C D(C M-\beta-C D)$, sulphated $\beta-C D$ $(S-\beta-C D)$ (Sigma-Aldrich, Taufkirchen, Germany), and sulphobutylter- $\beta-C D$ (SBE- $\beta-C D)$ (Cydex Pharmaceuticals, San Diego, CA, USA).

To determine the concentration of enantiomers in pharmaceutical products, Verapamil $80 \mathrm{mg}$ tablets (Sanofi Aventis, Paris, France) were used. The tablets were supplied by a local pharmacy.

\subsection{Instrumentation}

The experiments were performed on an Agilent 1600 CE system (Agilent Technologies, Waldbronn, Germany) equipped with a diode array detector (DAD). Chiral separations were performed using a short silica capillary (Agilent Technologies, Waldbronn, Germany) with a length of $30 \mathrm{~cm}$ (effective length $22 \mathrm{~cm}$ ) and an internal diameter of $50 \mu \mathrm{m}$. Electropherograms were recorded and processed using Chemstation 7.01 software (Agilent, Waldbronn, Germany).

The $\mathrm{pH}$ of the BGE solution was determined using a Terminal $740 \mathrm{pH}$ meter (Inolab, Munich, Germany).

For the verification of method performances, Microsoft Excel 365 software (Microsoft, Redmond, Washington, DC, USA) was used; the experimental design was carried out using Design Expert 7.0 software (State-Ease, Minneapolis, MN, USA).

To evaluate chiral interactions between TM- $\beta-C D$ and the VER enantiomers and the geometry of the complex, semi-empirical (PM3), and molecular mechanisms (MM+), calculations were performed using the HyperChem 8.0 (Hypercube, Gainesville, FL, USA). The starting geometries of the guest molecules, protonated according to their default protonation state at $\mathrm{pH}=7.0$, were constructed with the help of ChemBioOffice Ultra 11 software (CambridgeSoft, Cambridge, UK). The structure of TM- $\beta$-CD was creating adding methyl groups to $\beta-C D$, obtained from Cambridge Structural Database [21].

In our experiments of selecting the most stable conformation of the VER-TM $\beta-C D$ system, both the dimethoxyphenyl groups and the pentanenitrile moieties of the VER enantiomers were placed in the center of the CD cavity, at the wider and the narrower rim; then the guest molecule was completely rotated along the Z-axis in small increments with geometry optimization. The experiments were performed considering two other orientations of the guest molecules in relation to the TM $\beta-C D$,; with the VER molecule placed near the wider and narrow ring the CD molecule, alternatively. Therefore, eight configurations, for each VER enantiomer, were taken into consideration in searching for the most stable conformation. The complexes of TM- $\beta-C D$ with $R-V E R$ and $S$-VER, with stoichiometry 1:1, with the lowest energy obtained by MM+ calculations (PolakRibiere algorithm, RMS < 0.01) were fully optimized by the PM3 method in vacuum, with convergence limit SCF $=0.001$. The binding energy $\left(E_{\text {binding }}\right)$ of the complexes was calculated using the formula:

$$
E_{\text {binding }}=E_{\mathrm{TM}-\beta-\mathrm{CD}-\mathrm{VER}}-\left(E_{\mathrm{TM}-\beta-\mathrm{CD}}+E_{\mathrm{VER}}\right)
$$

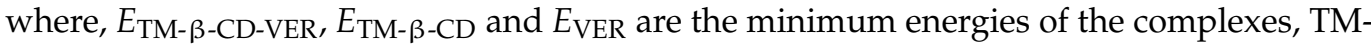
$\beta-C D$ and VER enantiomers, respectively. According to this equation, a negative value of $E_{\text {binding }}$ shows that the inclusion processes are energetically favorable, the highest negative value indicating the most stable complex.

\subsection{Electrophoretic Conditions}

The capillary conditioning before use was performed for $30 \mathrm{~min}$ with a $0.1 \mathrm{M} \mathrm{NaOH}$ solution, 15 min with purified water, and for another 15 min with the BGE solution. 
Between measurements, the capillary was preconditioned for 2 min with $0.1 \mathrm{M} \mathrm{NaOH}$ solution, purified water for $1 \mathrm{~min}$, and BGE solution for $2 \mathrm{~min}$.

Buffer solutions were prepared by dissolving the appropriate amount of its constituents in purified water. If necessary, the $\mathrm{pH}$ was adjusted by adding $1 \mathrm{M}$ phosphoric acid or $1 \mathrm{M} \mathrm{NaOH}$ solutions. Stock solutions of VER hydrochloride racemic mixture were prepared in methanol.

Before use, both buffer and sample solutions were filtered using a $0.45 \mu \mathrm{m}$ pore diameter filter, followed by ultrasonic degassing for $5 \mathrm{~min}$. The solutions were kept refrigerated when not in use.

Samples and standard solutions were hydrodynamically injected at the anodic end of the capillary, with detection at the cathode. The detection was carried out at 210, 230, and $280 \mathrm{~nm}$ wavelengths, based on the maximum absorption values of VER.

To evaluate analyte-chiral selector interactions, specific separation parameters were considered (separation factor and chiral resolution). The separation factor $(\alpha)$ was calculated as the ratio of the migration times of the two enantiomers, while the resolution was calculated following the formula: $R=2\left(t_{2}-t_{1}\right) /\left(w_{1}+w_{2}\right)$, where $t_{1}$ and $t_{2}$ represent the migration times and $\mathrm{w}_{1}$ and $\mathrm{w}_{2}$ the peak widths of the two enantiomers.

\subsection{Pharmaceutical Sample Preparation}

For the samples prepared from the pharmaceutical preparations, ten tablets were weighed, ground, and homogenized in a mortar. An amount of powder corresponding to the average mass of one tablet ( $80 \mathrm{mg}$ VER hydrochloride) was dissolved in methanol and homogenized on the ultrasonic bath for $5 \mathrm{~min}$. The solutions were brought to the appropriate concentrations with methanol before being introduced into the CE system. Electrophoretic conditions were the same as those used in the chiral analysis of the standard.

\section{Results}

\subsection{Preliminary Analysis}

VER, is a phenylalkylamine derivative, a basic compound, with a $\mathrm{pK}_{\mathrm{a}}$ value of 8.8 , being positively ionized in an acidic medium [11]. In order to determine the electrophoretic behavior of the analyte in an achiral system, the $\mathrm{pH}$ of the BGE solution was varied over a range of 2.5-9.0 (at four $\mathrm{pH}$ levels-2.5, 5.0, 7.0, and 9.0) using a $25 \mathrm{mM}$ phosphate $\mathrm{BGE}$. The migration times decreased when increasing the $\mathrm{pH}$ of the BGE solution. With increasing $\mathrm{pH}$ values of $\mathrm{BGE}$, VER migrated close to the electroosmotic flow (EOF), due to the low electrophoretic mobility of the analyte in basic BGEs.

A complex CD screening was used to establish the optimal CS. Natural and neutral derivatized CDs were added in the BGE solution in a $10 \mathrm{mM}$ concentration, while anionic derivatized ones were added in a $5 \mathrm{mM}$ concentration in order to avoid generating elevated current within the capillary. $\beta-\mathrm{CD}$ (at $\mathrm{pH} 2.5)$ and heptakis $2,3,6$-tri methoxy- $\beta-\mathrm{CD}$ (TM- $\beta$-CD) (at $\mathrm{pH} 2.5,5.0,7.0$ ) were the only CDs which showed chiral interaction with VER. The use of $\beta-C D$ generated longer migration times and lower separation resolution. Consequently, taking into consideration the chiral resolution values (initial $\mathrm{R}=0.70$ ) and the shape and amplitude of the peaks, TM- $\beta-C D$ was chosen as the optimal CD to be used for the following determinations at a $\mathrm{pH}$ value of 5.0.

TM- $\beta-C D$ is a neutral derivatized $C D$ with no electrophoretic mobility; therefore, the electrophoretic mobility of the VER-TM- $\beta$-CD complex will be defined by the ionization of VER.

\subsection{Method Optimization}

An initial OFAT optimization method was applied to characterize the influence of electrophoretic parameters on the chiral resolution and to identify the significant parameters influencing chiral separation.

The following parameters involved in the chiral separation process were varied: BGE $\mathrm{pH}, \mathrm{BGE}$ concentration, $\mathrm{CD}$ concentration, voltage, temperature, and injection pressure. 
Chiral resolution and enantiomers migration times were taken into consideration as analytical responses. The following analytical parameters were the starting point in method development: $50 \mathrm{mM}$ phosphate BGE, BGE $\mathrm{pH} 5.0,25 \mathrm{kV}$ voltage, $20^{\circ} \mathrm{C}$ temperature, and $50 \mathrm{mbar} / \mathrm{s}$ hydrodynamic injection. An overview of the results obtained following OFAT screening technique is presented in Table 1.

Table 1. OFAT screening results for CE chiral separation of VER.

\begin{tabular}{ccccccccc}
\hline $\mathbf{p H}$ & $\begin{array}{c}\text { BGE } \\
\text { Conc. } \\
{[\mathbf{m M}]}\end{array}$ & $\begin{array}{c}\text { CD } \\
\text { Conc. } \\
{[\mathbf{m M}]}\end{array}$ & $\begin{array}{c}\text { Voltage } \\
{[\mathbf{k V}]}\end{array}$ & $\begin{array}{c}\text { Temperature } \\
{\left[{ }^{\circ} \mathbf{C}\right]}\end{array}$ & $\begin{array}{c}\text { Injection } \\
\text { Pressure } \\
{[\mathbf{m b a r} / \mathbf{s}]}\end{array}$ & $\mathbf{R}$ & $\mathbf{t}_{\mathbf{1}}(\mathbf{m i n})$ & $\mathbf{t}_{\mathbf{2}}(\mathbf{m i n})$ \\
\hline 4 & 50 & 20 & 25 & 20 & 50 & 1.18 & 2.64 & 2.69 \\
\hline 5 & 50 & 20 & 25 & 20 & 50 & 1.25 & 2.53 & 2.63 \\
\hline 6 & 50 & 20 & 25 & 20 & 50 & 1.02 & 2.38 & 2.44 \\
\hline 5 & 25 & 20 & 25 & 20 & 50 & 1.08 & 2.43 & 2.50 \\
\hline 5 & 100 & 20 & 25 & 20 & 50 & 1.17 & 3.45 & 3.56 \\
\hline 5 & 50 & 10 & 25 & 20 & 50 & 0.65 & 2.15 & 2.20 \\
\hline 5 & 50 & 15 & 25 & 20 & 50 & 0.85 & 2.45 & 2.51 \\
\hline 5 & 50 & 20 & 20 & 20 & 50 & 1.34 & 3.70 & 3.80 \\
\hline 5 & 50 & 20 & 30 & 20 & 50 & 0.96 & 1.82 & 1.89 \\
\hline 5 & 50 & 20 & 25 & 15 & 50 & 1.34 & 2.88 & 3.00 \\
\hline 5 & 50 & 20 & 25 & 25 & 50 & 1.08 & 2.47 & 2.55 \\
\hline 5 & 50 & 20 & 25 & 20 & 40 & 1.26 & 2.64 & 2.72 \\
\hline 5 & 50 & 20 & 25 & 20 & 30 & 1.28 & 2.72 & 2.80 \\
\hline
\end{tabular}

TM- $\beta$-CD concentration was varied in the range of $10-20 \mathrm{mM}$ observing an increase in chiral resolution but also in migration times with increasing CD concentration. Higher concentrations of $\mathrm{CD}$ were not used in order to avoid any solubilization issues of the CS in the aqueous BGE.

Increasing the BGE concentration (25-100 mM) resulted in a slight increase in chiral resolution but also in migration times. However, increasing BGE concentration will lead to an increase in current intensity generated in the capillary, which has a limiting effect on electrophoretic separations.

An increase in the value of the applied voltage $(20-30 \mathrm{kV})$ led to a slight decrease in chiral resolution and migration times. Decreasing the temperature $\left(15-25^{\circ} \mathrm{C}\right)$ led to a better resolution, but to an increase in migration times. Injection pressure (30-50 mbar) in-fluences mainly the amplitude of the peaks; migration times were slightly influenced, but the chiral resolution was higher when using short injection times (1 s).

Taking into consideration the results obtained by applying the OFAT preliminary optimization step, $\mathrm{CD}$ concentration, voltage, and temperature were identified to significantly interfere with the chiral separation of VER enantiomers and were further optimized using a DoE approach.

For further optimization, we chose a Box-Behnken design, a special type of three-level fractional factorial design, that allows the construction of both 1st and 2nd order mathematical models. The Box-Behnken design was chosen because it has certain advantages over full three-level factorial designs, consisting of a smaller number of experiments and a consequent reduction in optimization time and costs, by providing high efficiency [22].

The parameters considered to be significant were varied on three different levels $(-1,0$, and 1$)$ while the other parameters considered less significant were kept constant throughout the determination ( $50 \mathrm{mM}$ phosphate BGE, BGE $\mathrm{pH}$ 5.0, and injection pressure/time $50 \mathrm{mbar} / \mathrm{s}$ ).

Therefore, in the experimental design the following factors were varied: factor ACD concentration $(18,20,22 \mathrm{mM})$, factor $B$-temperature $\left(15,20,25^{\circ} \mathrm{C}\right)$, and factor $\mathrm{C}$ voltage $(15,20,25 \mathrm{kV})$, the values being chosen based on the results obtained in the 
preliminary analysis. Two analytical responses were taken into consideration: response $1-$ migration time of the second enantiomer, and response 2-chiral resolution. The software generated a matrix of 17 experiments in which the selected factors were varied arbitrarily with experiments with central values for each experimental factor. The Box-Behnken experimental design matrix and the analytical responses are presented in Table 2.

Table 2. Box-Behnken experimental design for CE chiral separation of VER (Factor A-CD concentration, Factor B - voltage, Factor C - temperature, Response 1-migration time of the second enantiomer, and Response 2-resolution).

\begin{tabular}{cccccc}
\hline Run No & Factor A & Factor B & Factor C & Response 1 & Response 2 \\
\hline 1 & 20 & 25 & 15 & 3.61 & 1.29 \\
\hline 2 & 20 & 20 & 20 & 3.94 & 1.35 \\
\hline 3 & 18 & 20 & 25 & 3.34 & 1.02 \\
\hline 4 & 18 & 20 & 15 & 3.95 & 1.14 \\
\hline 5 & 20 & 20 & 25 & 3.77 & 1.28 \\
\hline 6 & 22 & 15 & 20 & 4.94 & 1.52 \\
\hline 7 & 20 & 20 & 20 & 4.02 & 1.4 \\
\hline 8 & 18 & 15 & 20 & 4.36 & 1.16 \\
\hline 9 & 20 & 20 & 20 & 3.99 & 1.32 \\
\hline 10 & 18 & 25 & 20 & 3.12 & 1.08 \\
\hline 11 & 22 & 25 & 20 & 3.82 & 1.25 \\
\hline 12 & 20 & 20 & 20 & 3.96 & 1.3 \\
\hline 13 & 20 & 25 & 25 & 3.08 & 1.15 \\
\hline 14 & 22 & 20 & 25 & 4.25 & 1.28 \\
\hline 15 & 20 & 15 & 25 & 4.48 & 1.32 \\
\hline 16 & 22 & 20 & 15 & 4.82 & 1.48 \\
\hline 17 & 20 & 15 & 15 & 4.95 & 1.56 \\
\hline & & & & &
\end{tabular}

The analysis of variance (ANOVA) was performed on the experimental model with the main focus being to estimate the accessibility of the predicted model. Insignificant factors were removed one by one from the model in order to improve it and the regression equations were recalculated after each factor elimination accordingly. For migration time, the model was based on a linear function, while for chiral resolution, it was based on a quadratic function. The following regression models were obtained:

$$
\text { Migration time }(\min )=4.04+0.38 \times \mathrm{A}-0.64 \times \mathrm{B}-0.14 \times \mathrm{C}
$$

$$
\text { Resolution }(\mathrm{R})=1.34+0.14 \times \mathrm{A}-0.099 \times \mathrm{B}-0.042 \times \mathrm{C}-0.047 \times \mathrm{AB}-0.098 \times \mathrm{A}^{2}
$$

where $\mathrm{A}-\mathrm{CD}$ concentration, $\mathrm{B}-$ Voltage, $\mathrm{C}$-Temperature.

As it can be observed, all three factors studied proved to have a significant effect on both responses. In the case of the migration time, a simple linear regression model was obtained involving three terms, while in the case of the resolution, an interaction and a quadratic term was also included in the final equation, demonstrating the complexity of the relationship between the experimental parameters and responses.

The coefficient of determination, $R^{2}$, and its adjusted form, $R^{2}$ adj were used as performance indicators of the models. $\mathrm{R}^{2}$ is defined as the ratio of the explained variation to the total variation, and it can also be considered as a characteristic of the calculated model. The more $R^{2}$ approaches value 1 , it demonstrates that there is a high correlation and dependence between the experimental and the predicted values. Furthermore, $R^{2}$ adj accounts 
for the number of model terms determined, based on a specific number of experiments, reaching an optimum when additional terms do not represent further value to the model. For migration time, the $\mathrm{R}^{2}$ value was 0.9827 , in accordance with the $\mathrm{R}^{2}$ adj value of 0.9787 , while for resolution, $R^{2}$ value was 0.9609 , confirmed by the $R^{2}$ adj value of 0.9432 . In both cases, the $R^{2}$ and $R^{2}$ adj values indicate that the models can be used for navigation in the experimental domain.

Three-dimensional response surface plots were drawn to characterize the relationships between the significant factors and the analytical responses. These 3D models are made by plotting each analytical response against two factors, with the third factor remaining at a constant value as is presented in Figure 2.
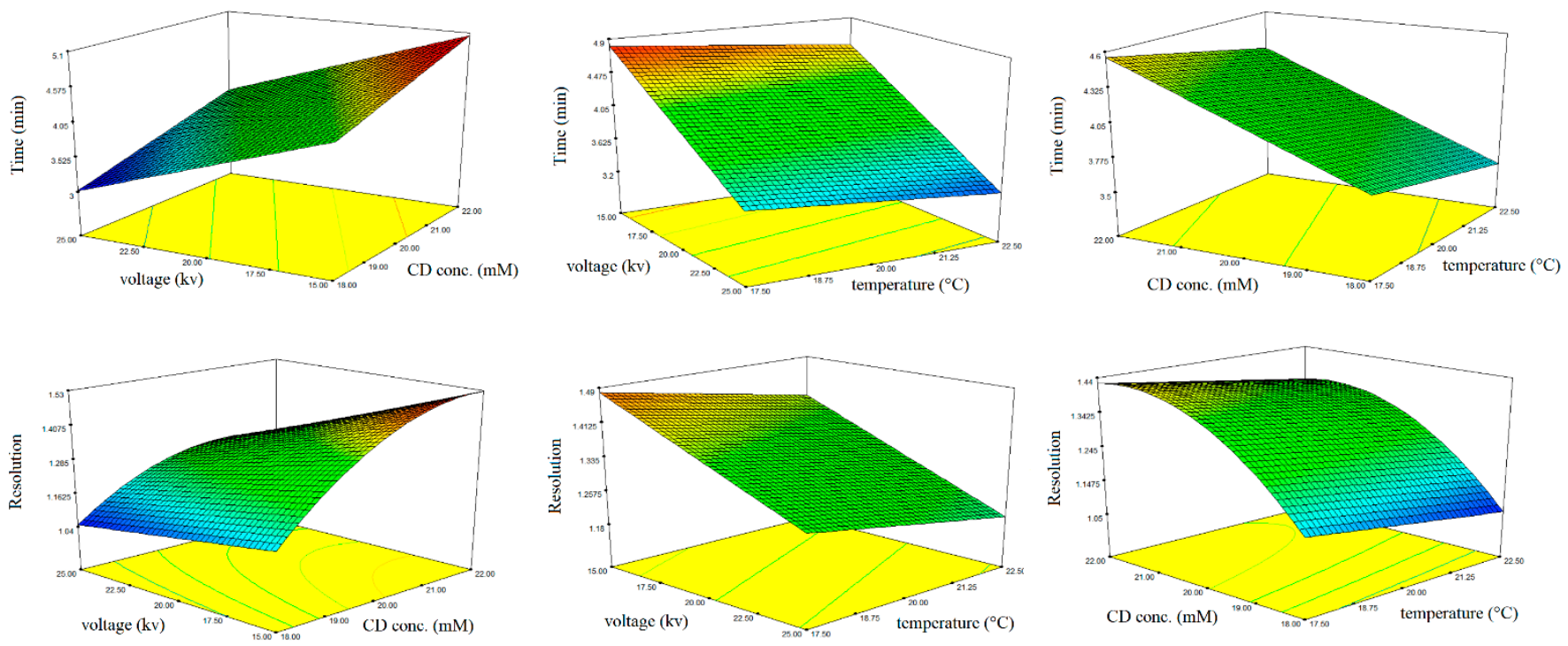

Figure 2. 3D response surface plots for resolution and migration time.

Desirability functions are usually designed in order to obtain different criteria, for example, to maximize, minimize, or target optimization of several analytical responses at the same time [23]. We used the numerical optimization function of the software, setting a maximum chiral resolution and a minimum migration time as objectives to be achieved. The factors were optimized with the same weight of priority. The optimal solution generated by the software was compared with the practical results obtained and both studied parameters, resolution and migration time, were similar.

The optimal conditions for the enantioseparation of VER enantiomers were as follows: $50 \mathrm{mM}$ phosphate BGE, BGE $\mathrm{pH}$ 5.0, $21 \mathrm{mM}$ TM- $\beta-\mathrm{CD}, 15^{\circ} \mathrm{C}$ temperature, $20 \mathrm{kV}$ voltage, and $50 \mathrm{mbar} / \mathrm{s}$ hydrodynamic injection. When applying the optimized analytical parameters, a chiral resolution of 1.58 , a selectivity factor $(\alpha)$ of 1.04 , and a short migration time of approximately $4 \mathrm{~min}$ were achieved. A representative electropherogram obtained by using the optimized conditions is presented in Figure 3.

Analytical parameters of the optimized method are presented in Table 3.

The migration order of the two enantiomers was determined, by spiking, adding the pure enantiomer $S$-VER, to a racemic VER sample, and was also based on the migration time of the pure enantiomer in the optimized chiral environment.

\subsection{Analytical Performance}

The analytical performance of the CE VER chiral separation method was tested in terms of precision and reproducibility (intra-day and inter-day), linearity, accuracy, and robustness (applying a Plackett-Burnam design). 


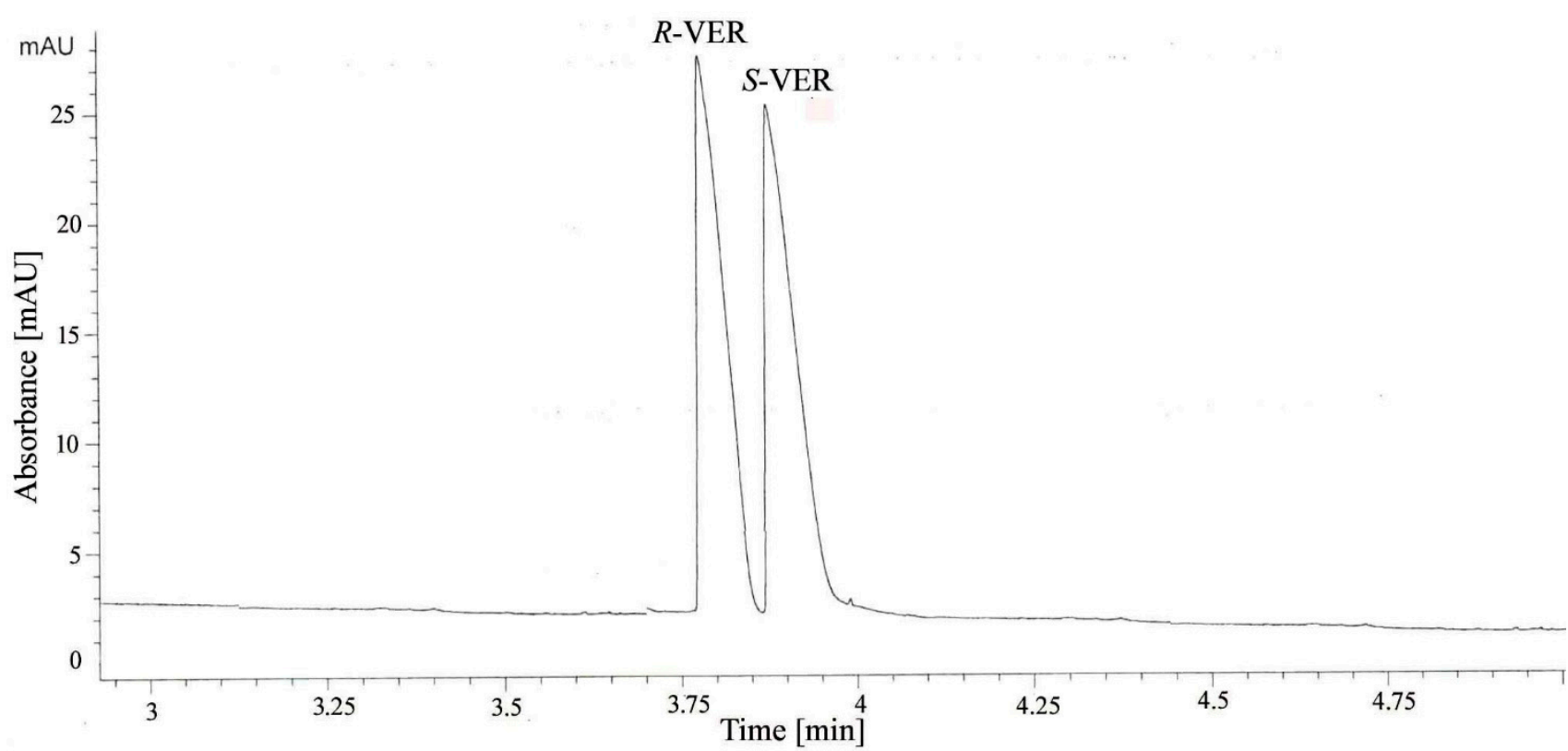

Figure 3. Chiral separation of VER using optimized condition (analytical conditions: $50 \mathrm{mM}$ phosphate BGE, pH 5.0, $21 \mathrm{mM}$ TM- $\beta$-CD, voltage $20 \mathrm{kV}$, temperature $15^{\circ} \mathrm{C}$, hydrodynamic injection $50 \mathrm{mbar} / \mathrm{s}$, UV detection $230 \mathrm{~nm}$, and VER concentration $0.15 \mathrm{mg} / \mathrm{mL}$ ).

Table 3. Analytical parameters of VFX chiral separation (analytical conditions: $50 \mathrm{mM}$ phosphate BGE, pH 5.0, $21 \mathrm{mM}$ TM- $\beta$-CD, voltage $20 \mathrm{kV}$, temperature $15^{\circ} \mathrm{C}$, hydrodynamic injection $50 \mathrm{mbar} / \mathrm{s}$, UV detection $230 \mathrm{~nm}$, and VER concentration $0.15 \mathrm{mg} / \mathrm{mL}$ ).

\begin{tabular}{cccccccc}
\hline Enantiomer & $\begin{array}{c}\text { Time } \\
{[\mathbf{m i n}]}\end{array}$ & Area & Height & Symmetry & $\begin{array}{c}\text { Nr Theoretical } \\
\text { Plates }\end{array}$ & Rs & $\boldsymbol{\alpha}$ \\
\hline R-VER & 3.82 & 90.05 & 25.77 & 0.13 & 18277 & - & - \\
\hline S-VER & 3.98 & 91.48 & 24.85 & 0.12 & 17545 & 1.58 & 1.04 \\
\hline
\end{tabular}

For the intra-day and inter-day precision evaluation, a racemic mixture of $0.15 \mathrm{mg} / \mathrm{mL}$ VER was injected six times on the same day and six times on three consecutive days, respectively. Relative standard deviations (RSDs) were calculated for migration time and peak area for the two enantiomers.

Calibration curves were obtained by measuring in triplicate standard solutions at nine different concentration levels over a chosen range: $0.015-0.3 \mathrm{mg} / \mathrm{mL}$. Correlation coefficients of over 0.99 indicate good linearity of the method.

Limits of detection (LOD) and quantification (LOQ) were determined as the ratio of the standard deviation of the regression equation and the slope of the regression equation multiplied by 3.3 and 10, respectively. The LOD and LOQ values were consistent with data from the literature when UV detection was used [12].

Recovery tests were performed in order to assess the accuracy of the method. The standard addition method was applied, as an appropriate quantity of VER powder from capsules was weighed and mixed with a known amount of the standard and each sample was analyzed three times. The good recovery values are an indication of accuracy.

The results obtained during the verification of analytical performance are summarized in Table 4.

In order to demonstrate the robustness of the method, a Plackett-Burnham design was applied, which is a two-level fractional factorial design model that allows the study of $\mathrm{N}-1$ experimental parameters in a number of $\mathrm{N}$ experiments [24]. The following five parameters were taken into consideration: BGE concentration $(45,50,55 \mathrm{mM}), \mathrm{CD}$ concentration (20.5, $21,21.5 \mathrm{mM})$, BGE pH $(4.5,5,5.5)$, voltage $(19,20,21 \mathrm{kV})$, and temperature $\left(14,15,16^{\circ} \mathrm{C}\right)$ while the analytical responses were the chiral resolution and the migration time of the 
second enantiomer. An overview of the Plackett-Burnham design is presented within Table 5. Based on the ANOVA statistical evaluation performed, it was concluded that no significant influence of any of the studied parameters was observed and the method robustness was confirmed.

Table 4. Analytical performance of the optimized method.

\begin{tabular}{|c|c|c|c|}
\hline \multicolumn{2}{|l|}{ VER } & $R$-VER & $S$-VER \\
\hline \multicolumn{4}{|c|}{ Precision } \\
\hline \multirow{2}{*}{$\begin{array}{l}\text { Intra-day precision (sample } \\
\text { concentration }=0.15 \mathrm{mg} / \mathrm{mL}, \mathrm{n}=6)\end{array}$} & RSD $\%$, migration time & 0.03 & 0.03 \\
\hline & RSD $\%$, peak area & 0.28 & 0.25 \\
\hline \multirow{2}{*}{$\begin{array}{l}\quad \text { Inter-day precision (sample } \\
\text { concentration }=0.15 \mathrm{mg} / \mathrm{mL}, \mathrm{n}=18 \text { ) }\end{array}$} & RSD $\%$, migration time & 0.05 & 0.06 \\
\hline & $\mathrm{RSD} \%$, peak area & 0.48 & 0.51 \\
\hline \multicolumn{4}{|c|}{ Accuracy (recovery $\% \pm$ SD) } \\
\hline \multicolumn{2}{|c|}{$0.15 \mathrm{mg} / \mathrm{mL}(\mathrm{n}=6)$} & $101.05 \pm 0.41$ & $99.55 \pm 0.63$ \\
\hline \multicolumn{2}{|c|}{$0.1 \mathrm{mg} / \mathrm{mL}(\mathrm{n}=6)$} & $100.58 \pm 1.02$ & $100.82 \pm 0.81$ \\
\hline \multicolumn{2}{|c|}{$0.075 \mathrm{mg} / \mathrm{mL}(\mathrm{n}=6)$} & $101.45 \pm 1.32$ & $99.87 \pm 1.24$ \\
\hline \multicolumn{4}{|c|}{ Linearity } \\
\hline \multicolumn{2}{|c|}{ Regression equation $(0.015-0.3 \mathrm{mg} / \mathrm{mL})$} & $y=275.42 x+0.0831$ & $y=278.1 x+0.4209$ \\
\hline \multicolumn{2}{|c|}{ Coefficient of correlation } & 0.996 & 0.994 \\
\hline \multicolumn{2}{|c|}{$\mathrm{LOD}(\mathrm{mg} / \mathrm{mL})$} & 0.0096 & 0.0092 \\
\hline \multicolumn{2}{|c|}{$\mathrm{LOQ}(\mathrm{mg} / \mathrm{mL})$} & 0.0291 & 0.0278 \\
\hline
\end{tabular}

Table 5. Plackett-Burnham design for robustness testing (Factor A-BGE concentration, Factor BCD concentration, Factor C-BGE pH, Factor D—voltage, Factor E-temperature, Response 1migration time, and Response 2-chiral resolution).

\begin{tabular}{cccccccc}
\hline $\mathbf{N r}$ & Factor A & Factor B & Factor C & Factor D & Factor E & Response 1 & Response 2 \\
\hline 1 & 50.00 & 21.00 & 5.00 & 20.00 & 15.00 & 3.49 & 1.49 \\
\hline 2 & 55.00 & 21.50 & 5.50 & 19.00 & 14.00 & 3.55 & 1.55 \\
\hline 3 & 45.00 & 21.50 & 5.50 & 19.00 & 16.00 & 3.52 & 1.46 \\
\hline 4 & 55.00 & 20.50 & 5.50 & 21.00 & 14.00 & 3.48 & 1.50 \\
\hline 5 & 45.00 & 20.50 & 4.50 & 21.00 & 14.00 & 3.44 & 1.47 \\
\hline 6 & 50.00 & 21.00 & 5.00 & 20.00 & 15.00 & 3.49 & 1.49 \\
\hline 7 & 55.00 & 21.50 & 4.50 & 19.00 & 14.00 & 3.54 & 1.53 \\
\hline 8 & 45.00 & 21.50 & 5.50 & 21.00 & 14.00 & 3.51 & 1.47 \\
\hline 9 & 55.00 & 20.50 & 4.50 & 19.00 & 16.00 & 3.52 & 1.53 \\
\hline 10 & 55.00 & 21.50 & 4.50 & 21.00 & 16.00 & 3.55 & 1.52 \\
\hline 11 & 50.00 & 21.00 & 5.00 & 20.00 & 15.00 & 3.49 & 1.48 \\
\hline 12 & 45.00 & 21.50 & 4.50 & 21.00 & 16.00 & 3.52 & 1.48 \\
\hline 13 & 55.00 & 20.50 & 5.50 & 21.00 & 16.00 & 3.51 & 1.50 \\
\hline 14 & 50.00 & 21.00 & 5.00 & 20.00 & 15.00 & 3.5 & 1.49 \\
\hline 15 & 45.00 & 20.50 & 5.50 & 19.00 & 16.00 & 3.45 & 1.47 \\
\hline 16 & 45.00 & 20.50 & 4.50 & 19.00 & 14.00 & 3.46 & 1.48 \\
\hline
\end{tabular}

The applicability of the method was verified through the determination of VER enantiomers from a pharmaceutical product, with a declared content of $80 \mathrm{mg}$ VER. The quantification of enantiomers was calculated with a calibration curve method. The absolute recovery of the enantiomers from pharmaceutical formulations was high, ranging from $98.5 \%$ and 
$100.3 \%$. Using our optimized electrophoretic method, the concentrations of the two enantiomers were determined and found to be in compliance with the quantities declared by the manufacturer, their enantiomer ratio being 1:1 (Table 6). Figure 4 shows a typical electropherogram of a pharmaceutical formulation sample. No peak that may have come from pharmaceutical formulation excipients interfered with the VER enantiomer separation.

Table 6. VER enantioselective analysis from pharmaceutical formulation.

\begin{tabular}{ccccc}
\hline \multirow{2}{*}{$\begin{array}{c}\text { Pharmaceutical } \\
\text { Product }\end{array}$} & \multicolumn{2}{c}{$\begin{array}{c}\text { Declared Enantiomers } \\
\text { Quantity }(\mathbf{m g})\end{array}$} & \multicolumn{2}{c}{$\begin{array}{c}\text { Found Enantiomer } \\
\text { Quantity }(\mathbf{m g}) \pm \text { SD }(\mathbf{n}=\mathbf{3})\end{array}$} \\
\cline { 2 - 5 } & $\boldsymbol{R}$-VER & S-VER & $\boldsymbol{R}$-VER & S-VER \\
\hline $\begin{array}{c}\text { Verapamil tablets } \\
(80 \mathrm{mg} \text { VER })\end{array}$ & 40 & 40 & $39.85 \pm 0.27$ & $39.72 \pm 0.32$ \\
\hline
\end{tabular}

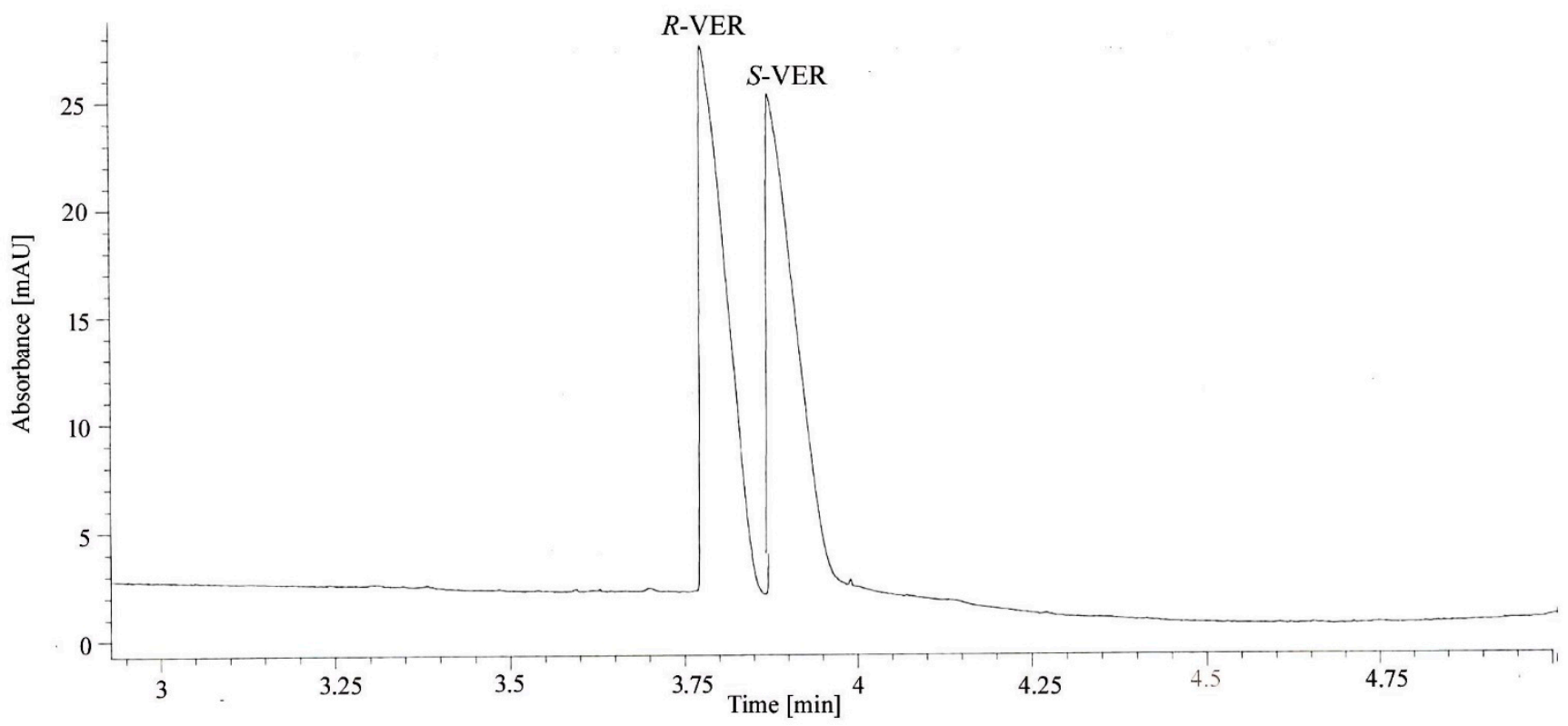

Figure 4. Chiral separation of VER enantiomers from pharmaceutical preparations (analytical conditions: $50 \mathrm{mM}$ phosphate BGE, pH 5.0, $21 \mathrm{mM}$ TM- $\beta-\mathrm{CD}$, voltage $20 \mathrm{kV}$, temperature $15^{\circ} \mathrm{C}$, hydrodynamic injection $50 \mathrm{mbar} / \mathrm{s}$, and UV detection $230 \mathrm{~nm}$ ).

\subsection{Molecular Modelling of VER-CD Complexes}

Molecular modelling methods were applied to obtain information on the interaction energy as well as preliminary data of the geometry of the inclusion complexes. According to PM3 results, both VER enantiomers formed stable complexes with the TM- $\beta-C D$. The energy minimized models for VER enantiomers and TM- $\beta-C D$ are presented in Figure 5. Regarding the geometry of the complexes, the pentanenitrile moiety of the VER is included in the TM- $\beta$-CDs cavity in the case of both enantiomers (Figure 6). Hydrogen bonding and van der Waals interactions stabilize the complex observed by analyzing the partial energies of the $E_{\text {binding }}$ and the distance between the atoms of the host and guest molecules.

Inclusion of $S$-VER into the studied CD $\left(E_{\text {binding }}-21.46 \mathrm{kcal} / \mathrm{mol}\right)$ is thermodynamically favorable over the $R$-VER complexation $\left(E_{\text {binding }}-16.83 \mathrm{kcal} / \mathrm{mol}\right)$, characterized by an energy difference of $4.63 \mathrm{kcal} / \mathrm{mol}$ according to the PM3 calculations, which conforms to the experimental migration order (spiking) of the VER enantiomers. In addition, the observed difference in the strength of the hydrogen bonding and van der Waals interactions between the complexes can cause the enantioselectivity of the separation. 


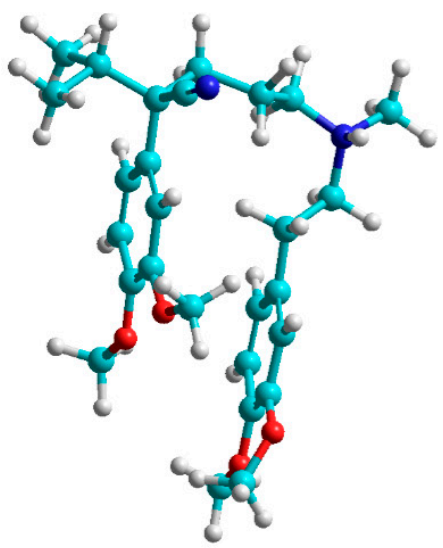

S.VER

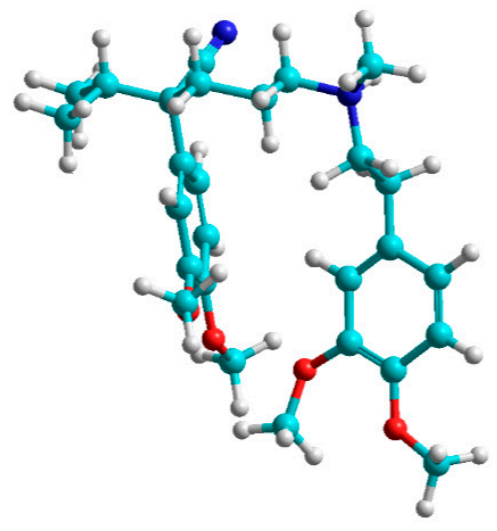

$R$-VER

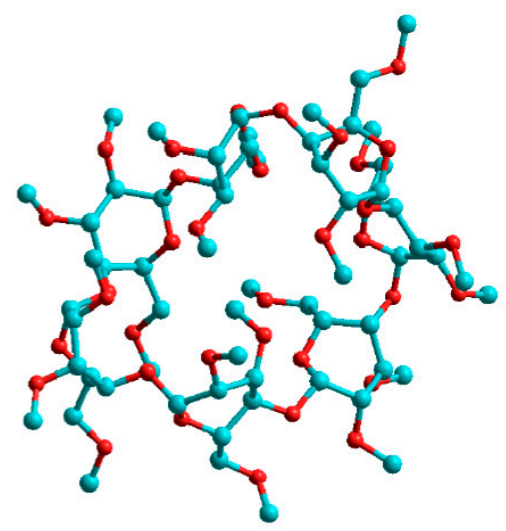

TM- $\beta-\mathrm{CD}$

Figure 5. Energy minimized models (PM3) of VER enantiomers and TM- $\beta-C D$.

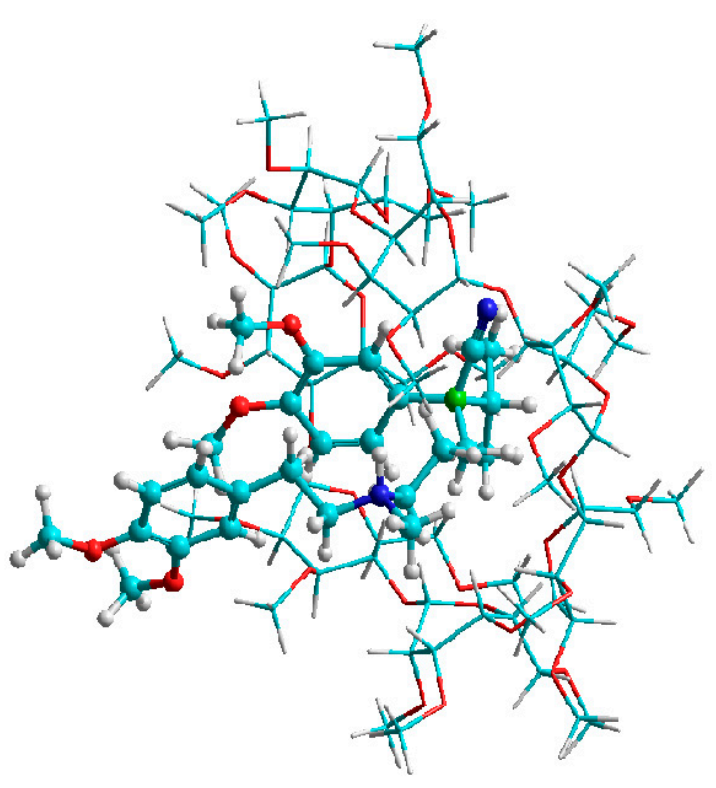

S-VER - TM- $\beta-C D$

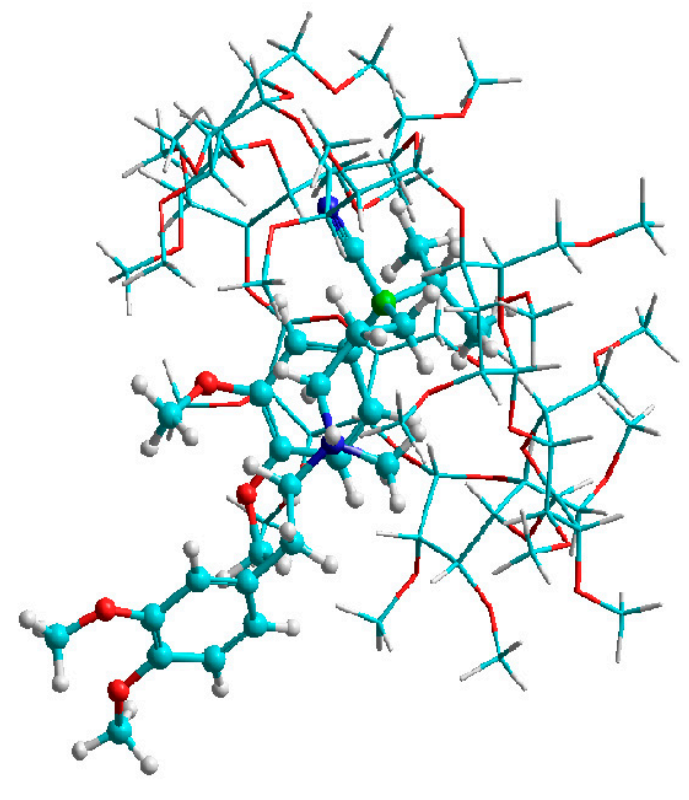

$R$-VER - TM- - -CD

Figure 6. Energy minimized models (PM3) of the VER-TM- $\beta$-CD complexes (the chiral carbon atom is marked in green; white is hydrogen, light blue is carbon, red is oxygen, and dark blue is nitrogen).

\section{Discussion}

A simple, rapid, and efficient method for the enantioseparation of VER via CE using $\mathrm{CD}$ as chiral selectors has been developed. Based on the preliminary $\mathrm{CD}$ screening at four different $\mathrm{pH}$ levels, TM- $\beta-\mathrm{CD}$, a derivatized neural $\mathrm{CD}$ at $\mathrm{pH} 5.0$, was chosen as the opti-mum CS.

For the analytical parameter optimization, we initially applied a univariate technique, whereby one parameter was varied individually while the others remained un-changed. After identifying the most important parameters on the enantioseparation (CD concentration, temperature, and voltage), a Box-Behnken DoE was applied to establish the optimal analytical conditions.

Based on the results obtained from the method optimization, chiral separation of VER enantiomers was achieved with a resolution of 1.58 and a migration time of approximately $4 \mathrm{~min}$, the order of migration being $R$-VER followed by $S$-VER. Probably $R$-VER forms a more stable complex with the CS than $S$-VER, and will migrate faster than its antipode. 
The analytical performance of the method was evaluated in terms of precision, reproducibility, linearity, accuracy, and robustness by applying a Plackett-Burnam DoE. The applicability of the method was verified by determining the ratio of the two enantiomers from a pharmaceutical product.

Applying molecular modelling calculations, it was established that the stability of the VER-CD inclusion complexes enables chiral discrimination and leads to differences in the migration times of the complexes. However, the theoretically calculated complexation energy difference $(4.6 \mathrm{kcal} / \mathrm{mol}) \mathrm{can}$ be interpreted as relatively small. The baseline resolution exceeding this thermodynamic mechanism of chiral separation may be due to the complementary effect of the electrophoretic mechanism (the difference in the effective mobility of enantiomer-selector complexes), representing a specific particularity of electromigration techniques [25].

Our CE method provides a good resolution in a short analysis time (4 min) if compared with other CE methods published previously in the literature. Dethy et al. [9] obtained the chiral separation of both VER and NVER in approximately 10 min with comparable resolution values using trimethyl- $\beta-C D$ as CS; Clothier \& Tomellini [10] obtained the chiral separation of VER and NVER in over $20 \mathrm{~min}$ by MEKC; Chankvetadze et al. [11] reported the enantioseparation of VER using TM- $\beta$-CD as CS in over 10 min, while Resztak \& Glowka [12] reported the enantioseparation of VER and NVER in approximately 8 min using the same TM- $\beta$-CD.

Compared with previously published CE methods for the separation of VER, our method uses DoE in method development, which allows the identification of the most important input factors, of input factor settings that lead to optimized output responses, and the elucidation of input factor interactions.

When compared with the more frequently used HPLC enantioseparation, our proposed CE method exhibits less sensitivity, but, nevertheless, delivers fast analysis time, high separation efficiency, and minimization of analyte and solvent use.

Author Contributions: Conceptualization, M.C.-D. and G.H.; methodology, M.C.-D. and G.H.; writing — original draft preparation, M.C.-D., G.H. and I.F.; writing—review and editing, M.C.-D., G.H., L.A.P. and H.K.; supervision, G.H.; project administration, M.C.-D.; funding acquisition, H.K. All authors have read and agreed to the published version of the manuscript.

Funding: This research was funded by a project collaboration between Medical and Pharmaceutical Section of the Transylvanian Museum Society and Faculty of Pharmacy of Semmelweis University Hungary (contract nr. 106.2/2020) (H.K.).

Institutional Review Board Statement: Not applicable.

Informed Consent Statement: Not applicable.

Data Availability Statement: Not applicable.

Conflicts of Interest: The authors declare no conflict of interest.

\section{References}

1. Brooks, W.H.; Guida, W.C.; Daniel, K.G. The significance of chirality in drug design and development. Curr. Top Med. Chem. 2011, 11, 760-770. [CrossRef]

2. Pepine, C.J.; Faich, G.; Makuch, R. Verapamil use in patients with cardiovascular disease: An overview of randomized trials. Clin. Cardiol. 1998, 21, 633-641. [CrossRef]

3. Abernethy, D.R.; Wainer, I.W.; Longstreth, J.A.; Andrawis, N.S. Stereoselective verapamil disposition and dynamics in aging during racemic verapamil administration. J. Pharmacol. Exp. Ther. 1993, 266, 904-911.

4. Gupta, S.K.; Atkinson, L.; Tu, T.; Longstreth, J.A. Age and gender related changes in stereoselective pharmacokinetics and pharmacodynamics of verapamil and norverapamil. Br. J. Clin. Pharmacol. 1995, 40, 325-331. [CrossRef]

5. Fanali, S.; Chankvetadze, B. Some thoughts about enantioseparations in capillary electrophoresis. Electrophoresis 2019, 40, 2420-2437. [CrossRef]

6. Bernardo-Bermejo, S.; Sánchez-López, E.; Castro-Puyana, M.; Marina, M.L. Chiral capillary electrophoresis. Trend Anal. Chem. 2020, 124, 115807. [CrossRef] 
7. Řezanka, P.; Navrátilová, K.; Řezanka, M.; Král, V.; Sýkora, D. Application of cyclodextrins in chiral capillary electrophoresis. Electrophoresis 2014, 35, 2701-2721. [CrossRef]

8. Fukuda, I.M.; Pinto, C.F.F.; Moreira, C.D.S.; Saviano, A.M.; Lourenço, F.R. Design of experiments (DoE) applied to pharmaceutical and analytical quality by design (QbD). Braz. J. Pharm. Sci. 2018, 54,1-16. [CrossRef]

9. Dethy, J.M.; De Broux, S.; Lesne, M.; Longstreth, J.; Gilbert, P. Stereoselective determination of verapamil and norverapamil by capillary electrophoresis. J. Chromatogr. B Biomed. Appl. 1994, 654, 121-127. [CrossRef]

10. Clothier, J.G.; Tomellini, S.A. Chiral separation of verapamil and related compounds using micellar electrokinetic capillary chromatography with mixed micelles of bile salt and polyoxyethylene ethers. J. Chromatogr. A 1996, 723, 179-187. [CrossRef]

11. Chankvetadze, B.; Burjanadze, N.; Pintore, G.; Strickmann, D.; Bergenthal, D.; Blaschke, G. Chiral recognition of verapamil by cyclodextrins studied with capillary electrophoresis, NMR spectroscopy, and electrospray ionization mass spectrometry. Chirality 1999, 11, 635-644. [CrossRef]

12. Resztak, M.; Główka, F.K. Stereoselective CZE method for analysis of verapamil and norverapamil in human plasma. Acta Pol. Pharm. 2013, 70, 395-401. [PubMed]

13. Hamidi, S.; Jouyban, A. Capillary electrophoresis with UV detection, on-line stacking and off-line dispersive liquid-liquid microextraction for determination of verapamil enantiomers in plasma. Anal. Methods 2015, 7, 5820-5829. [CrossRef]

14. Mohamed, N.A.L.; Kuroda, Y.; Shibukawa, A.; Nakagawa, T.; El Gizawy, S.; Askal, H.F.; El Kommos, M.E. Enantioselective binding analysis of verapamil to plasma lipoproteins by capillary electrophoresis-frontal analysis. J. Chromatogr. A 2000, 875, 447-453. [CrossRef]

15. Ha, P.T.T.; Sluyts, I.; Van Dyck, S.; Zhang, J.; Gilissen, R.A.; Hoogmartens, J.; Van Schepdael, A. Chiral capillary electrophoretic analysis of verapamil metabolism by cytochrome P450 3A4. J. Chromatogr. A 2006, 1120, 94-101. [CrossRef] [PubMed]

16. Asensi-Bernardi, L.; Martín-Biosca, Y.; Escuder-Gilabert, L.; Sagrado, S.; Medina-Hernández, M.J. In-line capillary electrophoretic evaluation of the enantioselective metabolism of verapamil by cytochrome P3A4. J. Chromatogr. A 2013, 1298, 139-145. [CrossRef] [PubMed]

17. Martín-Biosca, Y.; Escuder-Gilabert, L.; Sagrado, S.; Medina-Hernández, M.J. Enantioselective study on the biodegradation of verapamil and citalopram by chiral capillary electrophoresis. Separations 2021, 8, 29. [CrossRef]

18. Salido-Fortuna, S.; Greño, M.; Castro-Puyana, M.; Marina, M.L. Amino acid chiral ionic liquids combined with hydroxypropyl- $\beta$ cyclodextrin for drug enantioseparation by capillary electrophoresis. J. Chromatogr. A 2019, 1607, 460375. [CrossRef]

19. Ding, Y.; Zhu, X.; Lin, B. Study of interaction between drug enantiomers and serum albumin by capillary electrophoresis. Electrophoresis 1999, 20, 1890-1894. [CrossRef]

20. He, J.Y.; Shibukawa, A.; Zeng, M.; Amane, S.; Sawada, T.; Nakagawa, T. On-capillary sample preconcentration incorporated in chiral capillary electrophoresis. Anal. Sci. 1996, 12, 177-181. [CrossRef]

21. Groom, C.R.; Bruno, I.J.; Lightfoot, M.P.; Ward, S.C. The Cambridge Structural Database. Acta Crystallogr. B Struct. Sci. Cryst. Eng. Mater. 2016, 72, 171-179. [CrossRef]

22. Ferreira, S.C.; Bruns, R.E.; Ferreira, H.S.; Matos, G.D.; David, J.M.; Brandão, G.C.; da Silva, E.G.P.; Portugal, L.A.; dos Reis, P.S.; Souza, A.S.; et al. Box-Behnken design: An alternative for the optimization of analytical methods. Anal. Chim. Acta 2007, 597, 179-186. [CrossRef]

23. Candioti, L.V.; de Zan, M.M.; Cámara, M.S.; Goicoechea, H.C. Experimental design and multiple response optimization. Using the desirability function in analytical methods development. Talanta 2014, 124, 123-138. [CrossRef] [PubMed]

24. Ferreira, S.L.; Caires, A.O.; Borges, T.D.S.; Lima, A.M.; Silva, L.O.; dos Santos, W.N.L. Robustness evaluation in analytical methods optimized using experimental designs. Microchem. J. 2017, 131, 163-169. [CrossRef]

25. Chankvetadze, B. Contemporary theory of enantioseparations in capillary electrophoresis. J Chrom. A 2018, 1567, 2-25. [CrossRef] 